\title{
Computational Implementation of a New Multiphysics Model for Field Emission from CNT Thin Films
}

\author{
N. Sinha ${ }^{1}$, D. Roy Mahapatra ${ }^{2}$, R.V.N. Melnik ${ }^{3}$, and J.T.W. Yeow ${ }^{1}$ \\ ${ }^{1}$ Department of Systems Design Engineering, University of Waterloo, Waterloo, \\ Canada \\ ${ }^{2}$ Department of Aerospace Engineering, Indian Institute of Science, Bangalore, India \\ ${ }^{3} \mathrm{M}^{2} \mathrm{NeT}$ Lab, Wilfrid Laurier University, Waterloo, Canada \\ nsinha@engmail.uwaterloo.ca, droymahapatra@aero.iisc.ernet.in, \\ rmelnik@wlu.ca, jyeow@engmail .uwaterloo.ca
}

\begin{abstract}
Carbon nanotubes (CNTs) grown in a thin film have shown great potential as cathodes for the development several field emission devices. However, in modeling these important devices we face substantial challenges since the CNTs in a thin film undergo complex dynamics during field emission, which includes processes such as (1) evolution, (2) electromechanical interaction, (3) thermoelectric heating and (4) ballistic transport. These processes are coupled, nonlinear, and multiphysics in their nature. Therefore, they must be analyzed accurately from the stability and long-term performance view-point of the device. Fairly detailed physics-based models of CNTs considering some of these aspects have recently been reported by us. In this paper, we extend these models and focus on their computational implementation. All components of models are integrated at the computational level in a systematic manner in order to accurately calculate main characteristics such as the device current, which are particularly important for stable performance of CNT thin film cathodes in x-ray devices for precision biomedical instrumentation. The numerical simulations reported in this paper are able to reproduce several experimentally observed phenomena, which include fluctuating field emission current, deflected CNT tips and the heating process.
\end{abstract}

Keywords: carbon nanotubes, field emission, current density, phonon.

\section{Introduction}

Since their discovery in 1991 [1], a substantial interest has been shown for potential applications of carbon nanotubes (CNTs). As a result, numerous devices incorporating CNTs have been proposed. Although some of the applications of CNTs may be realized in distant future, their application as electron field emitters already show great potential today [2]. With significant improvement in their growth conditions, they rank among the best emitters that are currently available. These filed emitting cathodes have several advantages over the conventional thermionic cathodes: (i) current density from field emission would be 
orders of magnitude greater than in the thermionic case, (ii) a cold cathode would minimize the need for cooling, and (iii) a field emitting cathode can be miniaturized.

Field emission performance of an isolated CNT is found to be remarkable due to its structural integrity, high thermal conductivity and geometry. However, the situation becomes complex for cathodes comprising CNT thin films. In this case, individual CNTs are not always aligned normal to the substrate surface, which is due to electromechanical interaction among neighboring CNTs. Small spikes in the current have been observed experimentally 3. These can be attributed to change in gap between the CNT tip and the anode plate either due to elongation of CNTs under high bias voltage or due to degradation/fragmentation of CNTs. Also, there is a possibility of dynamic contact of pulled up CNT tips with the anode plate when the bias voltage is very high. In order to stabilize the collective field emission from a CNT based thin film, preferential breakdown of a small number of CNTs is achieved by increasing the bias voltage after initial exposure to certain low voltage [4. In addition, the coupled electron-phonon transport may produce temperature spikes. The temperature can significantly influence the electrical conductivity [5]. From the modeling aspect, this becomes a general case, but is very challenging. In this paper, we extend the results of [6]-7] and include some of these aspects, focusing on the device-level performance of CNTs in a thin film. A diode configuration is considered here, where the cathode contains a CNT thin film grown on a metallic substrate. The anode acts as the field emission current collector. A major concern in this work is the inherent coupling among (i) electromechanical forces causing deformation of CNTs and (ii) the ballistic electron-phonon transport. From a system perspective, such a detailed study proves to be useful in understanding the reason behind the experimentally observed fluctuation in device current, which is undesirable for applications such as precision x-ray generation biomedical devices.

\section{CNT Field Emission as a Multiphysics Process: The Development of a Mathematical Model}

The physics of field emission from a flat metallic substrate is fairly well understood. The current density $(J)$ due to field emission from a metallic surface is usually obtained by using the Fowler-Nordheim equation [8], which can be expressed as

$$
J=\frac{B E^{2}}{\Phi} \exp \left(-\frac{C \Phi^{3 / 2}}{E}\right),
$$

where $E$ is the electric field, $\Phi$ is the work function for the cathode material, and $B$ and $C$ are constants. In the CNT thin film problem, under the influence of sufficiently high voltage at ultra-high vacuum, the electrons emitted from the CNTs (mainly from the CNT tip region and emitted parallel to the axis of the tubes) reach the anode. Unlike the metallic emitters, here the surface of the cathode is not smooth. The cathode here consists of hollow tubes (CNTs) in curved shapes and with certain spacings. In addition, certain amount of impurities and carbon 
clusters may be present within the otherwise empty spaces in the film. Moreover, the CNTs undergo reorientation due to electromechanical interactions with the neighbouring CNTs during field emission. Analysis of these processes requires the determination of the current density by considering the individual geometry of the CNTs, their dynamic orientations and the variation in the electric field during electronic transport.

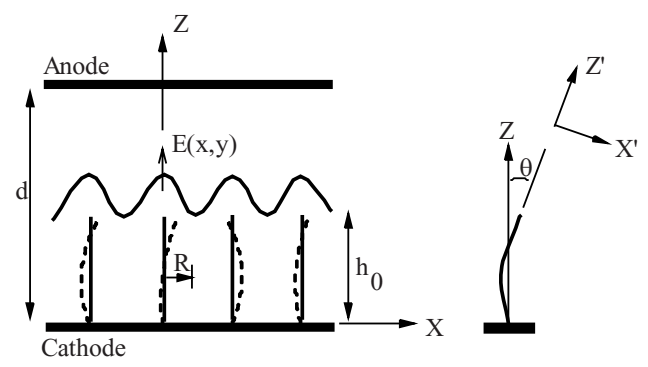

Fig. 1. CNT array configuration

In the present problem, we consider an array as shown in Fig. 1. A representative volume $\left(V_{\text {cell }}\right)$, which contains several CNTs with a prescribed distribution of their spacing at the substrate and random distribution of their curved shapes, is considered for the purpose of modeling. Furthermore, we discretize the CNT into several segments and nodes by treating each CNT as a $1 \mathrm{D}$ nanowire. At each node, we assign the quantities of interest, such as displacement, electron density, electric field and temperature. Next, governing equations involving these quantities of interest are derived in a systematic manner. An initial description of the thin film is given in terms of the tip angles and the curved shapes of the CNTs in $V_{\text {cell }}$, uniform conduction electron density of unstrained CNTs, a bias electric field and a reference temperature (temperature of the substrate). The phenomenological model of evolution of CNTs is given by four nonlinear coupled ordinary differential equations [6]. Based on this model, the rate of degradation of CNTs $v_{\text {burn }}$ is defined as

$$
v_{\text {burn }}=V_{\text {cell }} \frac{d n_{1}(t)}{d t}\left[\frac{s\left(s-a_{1}\right)\left(s-a_{2}\right)\left(s-a_{3}\right)}{n^{2} a_{1}^{2}+m^{2} a_{2}^{2}+n m\left(a_{1}^{2}+a_{2}^{2}-a_{3}^{2}\right)}\right]^{1 / 2},
$$

where $n_{1}$ is the concentration of carbon atoms in the cluster form in the cell, $a_{1}, a_{2}, a_{3}$ are lattice constants, $s=\frac{1}{2}\left(a_{1}+a_{2}+a_{3}\right), n$ and $m$ are integers $(n \geq|m| \geq 0)$. The pair $(n, m)$ defines the chirality of the CNT. Therefore, at a given time, the length of a CNT can be expressed as $h(t)=h_{0}-v_{\text {burn }} t$, where $h_{0}$ is the initial average height of the CNTs and $d$ is the distance between the cathode substrate and the anode (see Fig. 1).

The effective electric field component for field emission calculation in Eq. (1) is expressed as

$$
E_{z}=-e^{-1} \frac{d \mathcal{V}(z)}{d z},
$$


where $e$ is the positive electronic charge and $\mathcal{V}$ is the electrostatic potential energy. The total electrostatic potential energy can be expressed as

$$
\mathcal{V}(x, z)=-e V_{s}-e\left(V_{d}-V_{s}\right) \frac{z}{d}+\sum_{j} G(i, j)\left(\hat{n}_{j}-n\right),
$$

where $V_{s}$ is the constant source potential (on the substrate side), $V_{d}$ is the drain potential (on the anode side), $G(i, j)$ is the Green's function [9] with $i$ being the ring position, and $\hat{n}_{j}$ denotes the electron density at node position $j$ on the ring. The field emission current $\left(I_{\text {cell }}\right)$ from the anode surface associated with $V_{\text {cell }}$ of the film is obtained as

$$
I_{\text {cell }}=A_{\text {cell }} \sum_{j=1}^{N} J_{j},
$$

where $A_{\text {cell }}$ is the anode surface area and $N$ is the number of CNTs in the volume element. The total current is obtained by summing the cell-wise current $\left(I_{\text {cell }}\right)$. This formulation takes into account the effect of CNT tip orientations and one can perform statistical analysis of the device current for randomly distributed and randomly oriented CNTs. However, due to the deformation of the CNTs due to electromechanical forces, the evolution process requires a much more detailed treatment from the mechanics point of view.

Based on the studies reported in published literature [10-[12, it is reasonable to expect that a major contribution is by the Lorentz force due to the flow of electron gas along the CNT and the ponderomotive force due to electrons in the oscillatory electric field. The oscillatory electric field could be due to hopping of the electrons along the CNT surfaces and the changing relative distances between two CNT surfaces. In addition, the electrostatic force and the van der Waals force are also important. The net force components acting on the CNTs parallel to the $Z$ and the $X$ directions are calculated as $[7$

$$
\begin{aligned}
& f_{z}=\int\left(f_{l z}+f_{v s_{z}}\right) d s+f_{c_{z}}+f_{p_{z}}, \\
& f_{x}=\int\left(f_{l x}+f_{v s_{x}}\right) d s+f_{c_{x}}+f_{p_{x}} .
\end{aligned}
$$

where $f_{l}, f_{v s}, f_{c}$ and $f_{p}$ are Lorentz, van der Waals, Coulomb and ponderomotive forces, respectively, and $d s$ is the length of a small segment of CNTs.

Under the assumption of small strain and small curvature, the longitudinal strain $\varepsilon_{z z}$ (including thermal strain) and stress $\sigma_{z z}$ can be written as, respectively,

$$
\varepsilon_{z z}=\frac{\partial u_{z^{\prime} 0}^{(m)}}{\partial z^{\prime}}-r^{(m)} \frac{\partial^{2} u_{x^{\prime}}^{(m)}}{\partial z^{\prime 2}}+\alpha \Delta T\left(z^{\prime}\right), \quad \sigma_{z z}=E^{\prime} \varepsilon_{z z},
$$

where the superscript $(m)$ indicates the $m$ th wall of the multi walled CNT (MWNT) with $r^{(m)}$ as its radius, $u_{x^{\prime}}$ and $u_{z^{\prime}}$ are lateral and longitudinal dispacements of the oriented CNTs, $E^{\prime}$ is the effective modulus of elasticity of CNTs under consideration, $\Delta T\left(z^{\prime}\right)=T\left(z^{\prime}\right)-T_{0}$ is the difference between the absolute 
temperature $(T)$ during field emission and a reference temperature $\left(T_{0}\right)$, and $\alpha$ is the effective coefficient of thermal expansion (longitudinal). Next, by introducing the strain energy density, the kinetic energy density and the work density, and applying the Hamilton principle, we obtain the governing equations in $\left(u_{x^{\prime}}\right.$, $u_{z^{\prime}}$ ) for each CNT, which can be expressed as

$$
\begin{gathered}
E^{\prime} A_{2} \frac{\partial^{4} u_{x^{\prime}}}{\partial z^{\prime 4}}+\rho A_{0} \ddot{u}_{x^{\prime}}-\rho A_{2} \frac{\partial^{2} \ddot{u}_{x^{\prime}}}{\partial z^{\prime 2}}-f_{x^{\prime}}=0, \\
-E^{\prime} A_{0} \frac{\partial^{2} u_{z^{\prime} 0}}{\partial z^{\prime 2}}-\frac{E^{\prime} A_{0} \alpha}{2} \frac{\partial \Delta T\left(z^{\prime}\right)}{\partial z^{\prime}}+\rho A_{0} \ddot{u}_{z^{\prime} 0}-f_{z^{\prime}}=0,
\end{gathered}
$$

where $A_{2}$ is the second moment of cross-sectional area about $Z$-axis, $A_{0}$ is the effective cross-sectional area, and $\rho$ is the mass per unit length of CNT. We assume fixed boundary conditions $(u=0)$ at the substrate-CNT interface $(z=0)$ and forced boundary conditions at the CNT tip $(z=h(t))$.

By considering the Fourier heat conduction and thermal radiation from the surface of CNT, the energy rate balance equation in $T$ can be expressed as

$$
d Q-\frac{\pi d_{t}^{2}}{4} d q_{F}-\pi d_{t} \sigma_{S B}\left(T^{4}-T_{0}^{4}\right) d z^{\prime}=0,
$$

where $d Q$ is the heat flux due to Joule heating over a segment of a CNT, $q_{F}$ is the Fourier heat conduction, $d_{t}$ is the diameter of the CNT and $\sigma_{S B}$ is the Stefan-Boltzmann constant. First, the electric field at the nodes are computed and then all the governing equations are solved simultaneously at each time step and the curved shape $s\left(x^{\prime}+u_{x^{\prime}}, z^{\prime}+u_{z^{\prime}}\right)$ of each of the CNTs is updated. The angle of orientation $\theta$ between the nodes $j+1$ and $j$ at the two ends of segment $\Delta s_{j}$ is expressed as

$$
\theta(t)=\tan ^{-1}\left(\frac{\left(x^{j+1}+u_{x}^{j+1}\right)-\left(x^{j}+u_{x}^{j}\right)}{\left(z^{j+1}+u_{z}^{j+1}\right)-\left(z^{j}+u_{z}^{j}\right)}\right), \quad\left[\begin{array}{c}
u_{x}^{j} \\
u_{z}^{j}
\end{array}\right]=\left[\Gamma\left(\theta(t-\Delta t)^{j}\right)\right]\left[\begin{array}{c}
u_{x^{\prime}}^{j} \\
u_{z^{\prime}}^{j}
\end{array}\right],
$$

where $\Gamma$ is the usual coordinate transformation matrix which maps the displacements $\left(u_{x^{\prime}}, u_{z^{\prime}}\right)$ defined in the local $\left(X^{\prime}, Z^{\prime}\right)$ coordinate system into the displacements $\left(u_{x}, u_{z}\right)$ defined in the cell coordinate system $(X, Z)$.

\section{Computational Scheme, Results and Discussions}

A key characteristics is the device current, and in what follows we focus on the systematic integration of all the models at the computational level to calculate the device current. At a given time, the evolved concentration of carbon clusters due to the process of degradation and CNT fragmentation is obtained from the nucleation coupled model, which is modeled by assuming the degradation as a reverse process of growth (the nucleation theory has been used for the growth of CNTs [13). This information is then used in a time-incremental manner to describe the evolved state of the CNTs in the cells. At each time step, the net 


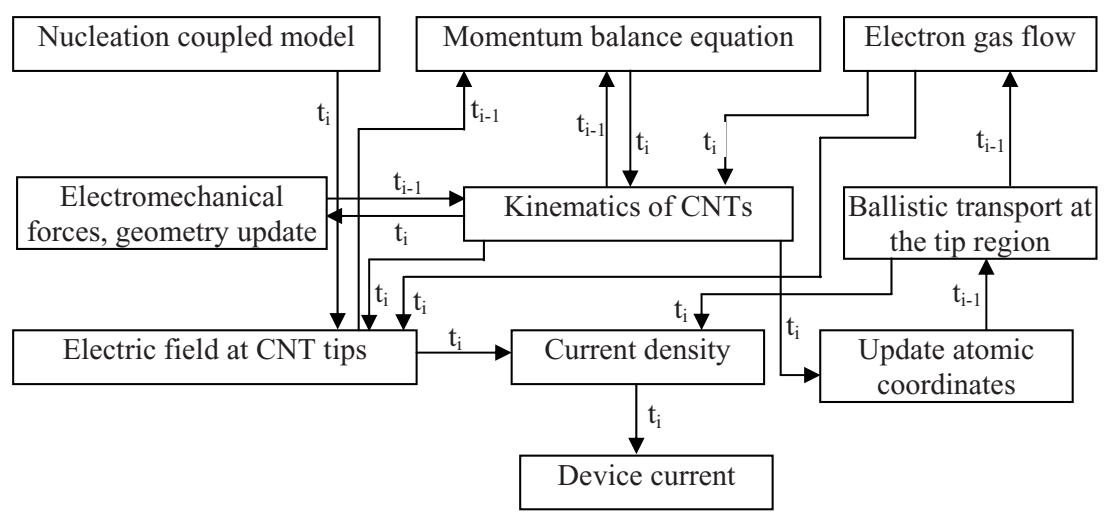

Fig. 2. Computational flowchart for calculating the device current

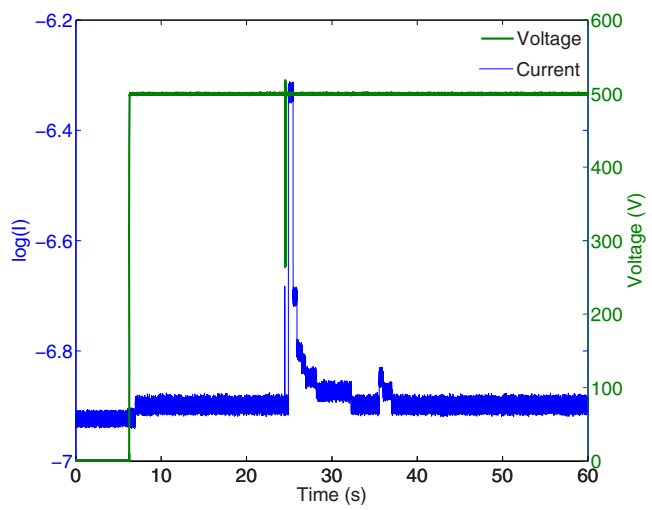

Fig. 3. Spikes in the field emission current at low bias voltage due to reorientation and pull-up of few CNTs

electromechanical force is computed using the momentum balance equation and equation for electron gas flow. Subsequently, the orientation angle of each CNT tip is obtained. Thereafter, we compute the electric field at the tip of CNTs at each time step. Finally, the current density and device current are calculated by employing Eq. (1). The computational flow chart for calculating the device current is shown in Fig. 2

The CNT film considered in this study consists of randomly oriented MWNTs. The film was grown on a stainless steel substrate. The film surface area (projected on anode) is $49.93 \mathrm{~mm}^{2}$ and the average height of the film (based on randomly distributed CNTs) is $12 \mu \mathrm{m}$. Actual experiments were carried out in a pressure controlled vacuum chamber and field emission current histories were measured under various DC bias voltages. Fig. 3 shows the occurence of current spikes at 


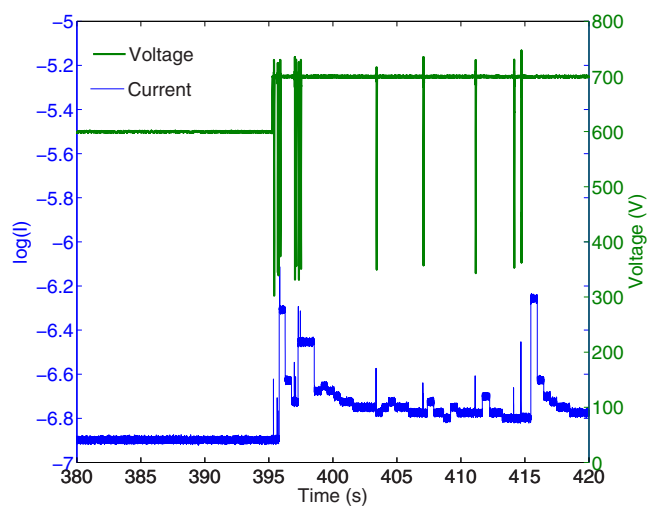

Fig. 4. Fluctuation of field emission current from a baked sample having vertically aligned CNTs

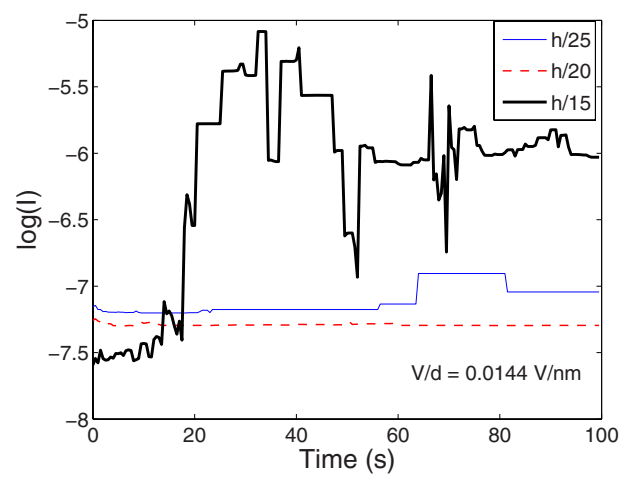

Fig. 5. Field emission current histories for various initial tip defelctions under a bias voltage of $500 \mathrm{~V}$

a voltage of $500 \mathrm{~V}$, which indicates that few CNTs are emitting heavily and are pulled up towards anode. More spikes are observed as bias voltage is increased to $700 \mathrm{~V}$ (see Fig. (4). In the simulation and analysis, the constants $\mathrm{B}$ and $\mathrm{C}$ in Eq. (1) were taken as $B=\left(1.4 \times 10^{-6}\right) \times \exp \left((9.8929) \times \Phi^{-1 / 2}\right)$ and $C=6.5 \times 10^{7}$, respectively [14. The initial height distribution $h$ and the orientation angle $\theta$ were randomly distributed. The electrode gap $(d)$ was maintained at $34.7 \mu \mathrm{m}$. The orientation of CNTs was parametrized in terms of the upper bound of the CNT tip deflection (denoted by $h_{0} / m^{\prime}, m^{\prime}>>1$ ). Several computational runs were performed and the output data were averaged out at each sampling time step. Figures [5] show the simulated current histories at different tip deflections and at different bias voltages. Following observations have been made from the 


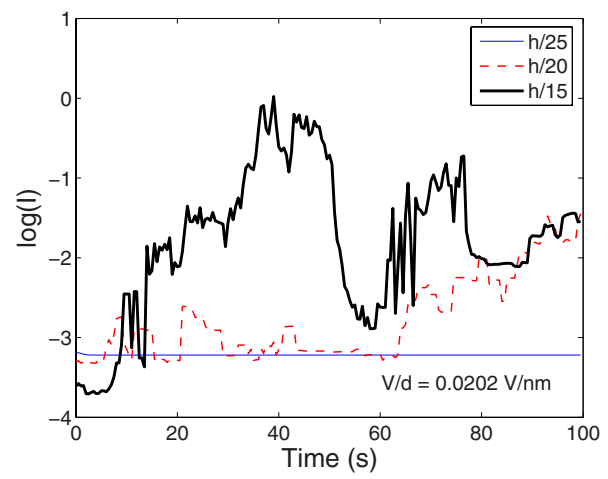

Fig. 6. Field emission current histories for various initial tip defelctions under a bias voltage of $700 \mathrm{~V}$

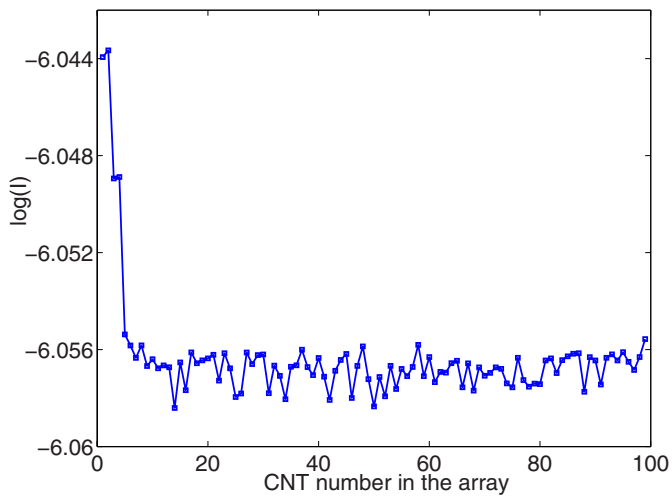

Fig. 7. Current at the CNT tips at $\mathrm{t}=100 \mathrm{~s}$ of field emission

results: (i) at a constant bias voltage, as the initial state of deflection of the CNTs increases (from $h_{0} / 25$ to $h_{0} / 20$ ), the average current reduces until the initial state of deflection becomes large enough $\left(h_{0} / 15\right)$ that the electrodynamic interaction among CNTs produces sudden pull in the deflected tips towards the anode resulting in current spikes; (ii) the amplitude factor of current spikes at higher bias is of the order of $\sim 10^{3}$. On the other hand, the trend indicates current spikes with an amplitude factor of $\sim 10^{2}$ for lower bias voltage. Figure 7 shows the tip current distribution at $t=100 \mathrm{~s}$ for an array of $100 \mathrm{CNTs}$. After calculating strain from Eq. (8)), corresponding changes in the bandgap along the CNT length were calculated using tight binding formulation for bandstructure as a function of strain [15. The value of Young's modulus used for the calculation was $0.27 \mathrm{TPa}$. The strained energy bandgap along the length of CNT is shown in Fig 8 . The unstrained bandgap value was found to be $3.0452 \mathrm{eV}$. As evident from 


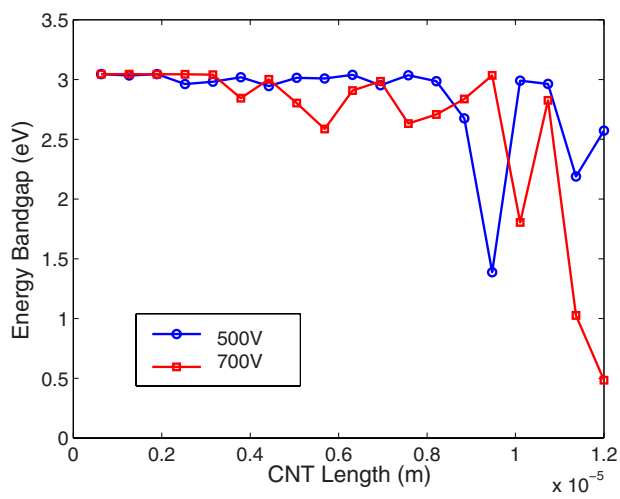

Fig. 8. Cross-sectional energy bandgap distribution along the CNT at 500V and 700V

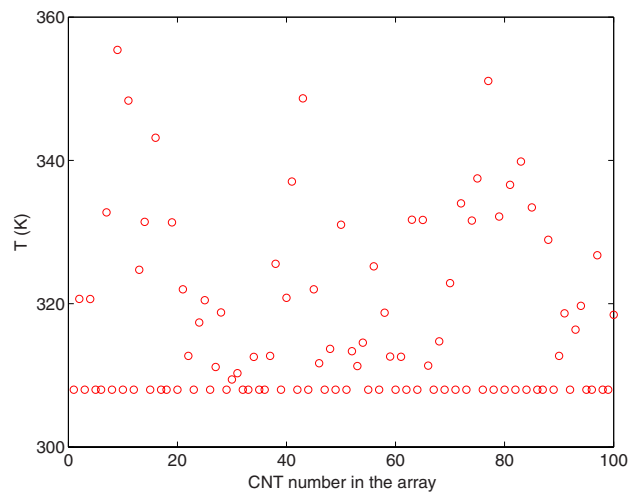

Fig. 9. Maximum temperature of CNT tips during $100 \mathrm{~s}$ of field emission

Fig. 8, as the strain increases, the energy bandgap decreases. In Fig. 9, maximum tip temperature distribution on the 100 CNTs during field emission over 100s duration is plotted. The maximum temperature rises up to approximately $358 \mathrm{~K}$.

\section{Concluding Remarks}

In this paper, a new multiphysics model has been proposed, which incorporates nonlinearities and coupling related to electrodynamic, mechanical and thermodynamic phenomena during the process of field emission. This model handles several complexities at the device scale and helps in understanding the fluctuation in the device current. Using the developed computational scheme, we were able to capture the transients in the field emission current, which have been 
observed in actual experiments. This model can be useful in designing CNT thin film cathodes having a stable field emission current and without compromising their lifetime.

\section{References}

1. Iijima, S.: Helical tubules of graphitic carbon. Nature 354, 56-58 (1991)

2. Bonard, J.M., Salvetat, J.P., Stockli, T., Forro, L., Chatelain, A.: Field emission from carbon nanotubes: perspectives for applications and clues to the emission mechanism. Appl. Phys. A 69, 245-254 (1999)

3. Bonard, J.M., Klinke, C., Dean, K.A., Coll, B.F.: Degradation and failure of carbon nanotube field emitters. Phys. Rev. B 67, 115406 (2003)

4. Siedel, R.V., Graham, A.P., Rajashekharan, B., Unger, E., Liebau, M., Duesberg, G.S., Kreupl, F., Hoenlein, W.: Bias dependence and electrical breakdown of small diameter single-walled carbon nanotubes. J. Appl. Phys. 96, 6694-6699 (2004)

5. Huang, N.Y., She, J.C., Chen, J., Deng, S.Z., Xu, N.S., Bishop, H., Huq, S.E., Wang, L., Zhong, D.Y., Wang, E.G., Chen, D.M.: Mechanism responsible for initiating carbon nanotube vacuum breakdown. Phys. Rev. Lett. 93, 75501 (2004)

6. Sinha, N., Roy Mahapatra, D., Yeow, J.T.W., Melnik, R.V.N., Jaffray, D.A.: Carbon nanotube thin film field emitting diode: understanding the system response based on multiphysics modeling. J. Compu. Theor. Nanosci. 4, 535-549 (2007)

7. Sinha, N., Roy Mahapatra, D., Sun, Y., Yeow, J.T.W., Melnik, R.V.N., Jaffray, D.A.: Electromchanical interactions in a carbon nanotube based thin film field emitting diode. Nanotechnology 19(1-12), 25701 (2008)

8. Fowler, R.H., Nordheim, L.: Electron emission in intense electric field. Proc. R. Soc. Lond. A 119, 173-181 (1928)

9. Svizhenko, A., Anantram, M.P.: Effect of scattering and contacts on current and electrostatics in carbon nanotubes. Phys. Rev. B 72, 85430 (2005)

10. Slepyan, G.Y., Maksimenko, S.A., Lakhtakia, A., Yevtushenko, O., Gusakov, A.V.: Electrodynamics of carbon nanotubes: dynamic conductivity, impedance boundary conditions, and surface wave propagation. Phys. Rev. B 60, 17136-17149 (1999)

11. Glukhova, O.E., Zhbanov, A.I., Torgashov, I.G., Sinistyn, N.I., Torgashov, G.V.: Ponderomotive forces effect on the field emission of carbon nanotube films. Appl. Surf. Sci. 215, 149-159 (2003)

12. Ruoff, R.S., Tersoff, J., Lorents, D.C., Subramoney, S., Chan, B.: Radial deformation of carbon nanotubes by van der Waals forces. Nature 364, 514-516 (1993)

13. Watanabe, T., Notoya, T., Ishigaki, T., Kuwano, H., Tanaka, H., Moriyoshi, Y.: Growth mechanism for carbon nanotubes in a plasma evaporation process. Thin Solid Films 506/507, 263-267 (2006)

14. Huang, Z.P., Tu, Y., Carnahan, D.L., Ren, Z.F.: Field emission of carbon nanotubes. Encycl. Nanosci. Nanotechnol. 3, 401-416 (2004)

15. Yang, L., Anantram, M.P., Han, J., Lu, J.P.: Band-gap change of carbon nanotubes: effect of small uniaxial strain and torsion strain. Phys. Rev. B 60, 13874-13878 (1999) 